\title{
An Automatic Optimal Course Recommendation Method for Online Math Education Platforms Based on Bayesian Model
}

\author{
https://doi.org/10.3991/ijet.v16i13.24039 \\ Yongyan Fan, Jing Zhang \\ Cangzhou Normal University, Cangzhou, China \\ Dingli Zu ${ }^{\bowtie}$, Hongyu Zhang \\ Chengde Petroleum College, Chengde, China \\ dttongcz@163.com
}

\begin{abstract}
Online education platforms inject new vitality into the field of education, and greatly improves the accessibility to high-quality education resources. However, the current online education platforms do not support independent course selection based on personal preferences. To solve this problem, this paper designs an automatic recommendation method of optimal courses for online math education platforms based on Bayesian model. The results show that the Bayesian model can simulate the causal relationship between real-world affairs by building a graphic model based on the graph theory and the probability theory; the model can effectively merge priori and posteriori information, and encode the causality between knowledge points; the model clearly outshines user-based collaborative filtering model, term-based collaborative filtering model, and SlopeOne model, and achieves a stable accuracy rate in automatic recommendation of courses. The research provides an empirical evidence to the improvement and innovation of professional online math course platforms.
\end{abstract}

Keywords - Online education platform, bayesian model, independent course selection, automatic recommendation, score prediction

\section{Introduction}

In the era of Internet+, the development of education is greatly promoted by communication and Internet technologies, and this has provided a new path for the comprehensive education reform in China [1]. In recent years, many foreign universities and colleges have tried to use the Internet to assist their teaching, now, the development momentum of such Internet-aided teaching is getting better and better, and has become an indispensable means of higher education [2, 3]. At present, online education has become the trend of future education, and the online education platforms can provide students with good venues for their autonomous learning [4]. 
In online education, course selection is a very important teaching management task. In order to achieve the training goals of modern talents, colleges and universities in China have carried out large-scale education system reforms, they have set more disciplines and courses, and students can choose their courses freely [5, 6]. Automatic course selection systems can realize active course recommendation that meets the individual needs of students [7]. The Bayesian model established based on Bayesian statistics is a reasonable combination of the Bayesian graph theory and the Bayesian method $[8,9]$. According to the different perspectives of the Bayesian network, the Bayesian network structure learning methods can be divided into two types, namely the methods based on conditional independence, and the methods based on scores [10]. The Bayesian model can be regarded as a structure containing the distribution of joint probabilities between attributes, through learning and scoring, the Bayesian network model that has the best data fits can be determined [11, 12]. Now, the Bayesian networks and models have been widely used in various fields such as information fusion, prediction, and business and intelligent robots [13, 14].

For online education platforms, computers are the bridge for learners to access to the digital courses on the Internet; in the context of the development of Internet and information technologies, they provide students with a new education mode that is different from the traditional classroom lecturing, and they emphasize on the form of learning [15]. Online courses are the educational resources shared on the online learning platforms, and they can promote the sharing of university courses on the Internet $[16,17]$. From the perspective of students, some studies found that whether the students have online course experience or not only affects the students' original recognition, it won't change their opinions [18]. Based on the Bayesian model, this paper attempts to explore an automatic optimal course recommendation method for online math education platforms, in the hopes of providing an evidence for the optimization and innovation of online course platforms of the math major.

\section{Theoretical Research}

\subsection{An overview of online education platforms}

Online education platforms are a kind of teaching method that delivers knowledge to students in a way that is different from the traditional face-to-face classroom lectures in the context of well-developed Internet and information technologies, they emphasize more on the form of learning [19]. Students use the online education platforms to conduct online learning, they can determine the learning venue and time by themselves, and such platforms can satisfy the individual learning needs of learners $[20,21]$. For an online education platform, the users include system administrators, teacher users, student users, and visitors [22]. The system administrators are in charge of reviewing the courses that have been applied for and managing the user rights of teachers and students in video uploads and recommendations, homework submission, and leaving messages and comments, etc. [23, 24]. Teachers have the rights of creating courses, uploading teaching materials, downloading homework in batches, com- 
menting, browsing comments, and recommending course videos, etc. [25]. Students can retrieve and study courses according to their preferences and needs; after completing and submitting their homework, they can view and comment on the homework of their peers, moreover, and they can also recommend the course videos they like [26, 27].

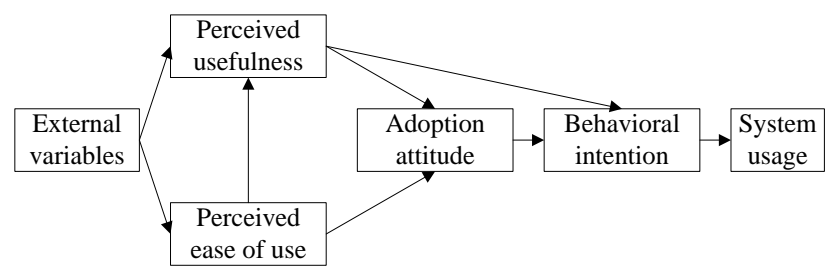

Fig. 1. Technology acceptance model

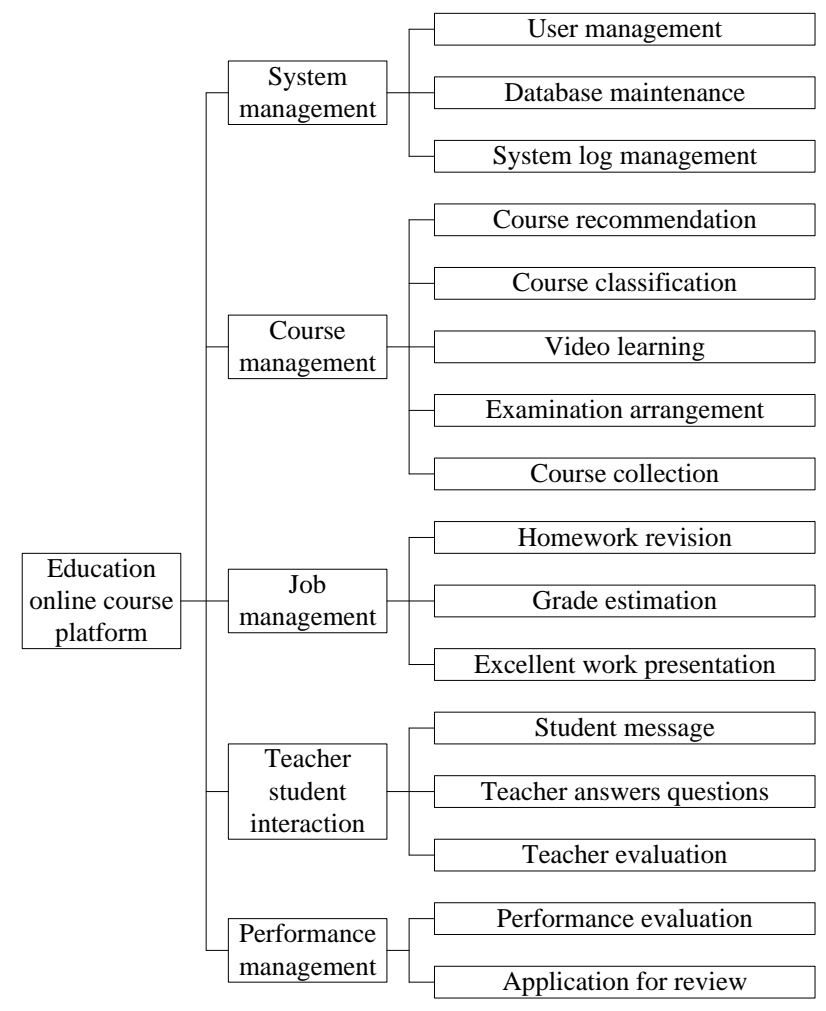

Fig. 2. Function modules of the online education platform

The technology acceptance model determines two variables that can affect certain user behaviors, namely the perceived usefulness, and the perceived ease of use, this model can be applied to research on the acceptance of information technologies and systems by various users [28]. Figure 1 shows the structure of the technology ac- 
ceptance model. Some scholars have founded during their research on online education platforms that the perceived ease of use is positively related to adoption attitude $[29,30]$. Figure 2 gives the function modules of the proposed online education platform; the five modules are: system management, course management, job management, teacher-student interaction, and performance management. The course management module includes five parts: course recommendation, course classification, video learning, examination arrangement and course collection [31]. In terms of the overall functions of the system, the platform can well satisfy the mixed learning requirements of multiple disciplines at the same time, and meet students' demands in autonomous and personalized learning, and fast course identification and selection [32].

\subsection{About the Bayesian model}

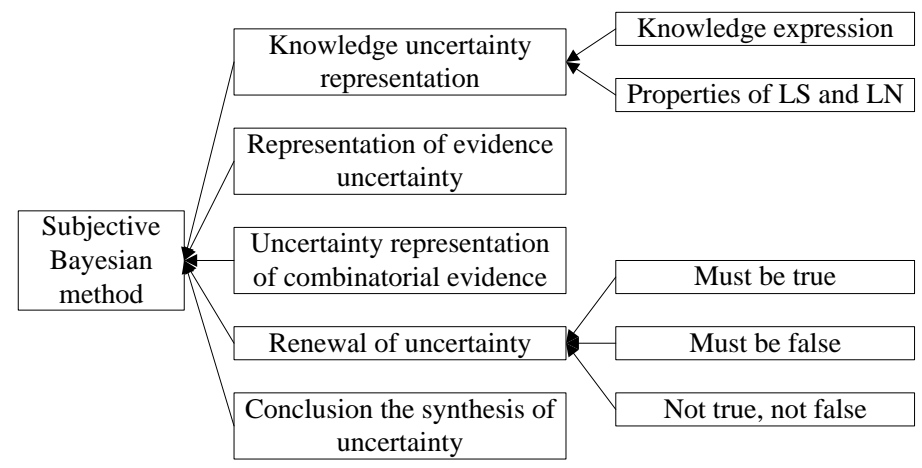

Fig. 3. Evaluation structure of the knowledge-point Bayesian network of the subjective Bayesian method

Personalized recommendation is the kernel technology for course selection systems. It has two attributes: the degree of automation and the degree of durability. Collaborative filtering is a commonly used automatic recommendation algorithm for course selection. The characteristic of the algorithm is to calculate the similarity between users based on the history information of the system and obtain users with similar interests, the algorithm can handle unstructured complex objects and has no special requirements for the objects being recommended. When dealing with data that contain a lot of interactions between the variables to be identified and the variables to be predicted, the Bayesian networks are usually adopted, they are additive, have no strict requirements for prior statistical assumptions, and can easily discover knowledge and structure from the massive and complex data, etc. The Bayesian model can effectively merge priori and posteriori information, and encode the causality between knowledge points; by continuously adding information of new evidences, it can timely update the evaluation and judge the course selection. In actual applications, the powerful reasoning and prediction ability of the Bayesian networks can be taken as a solid mathematical basis and this has ensured the credibility of the results. Figure 
3 shows the evaluation structure of the knowledge-point Bayesian network of the subjective Bayesian method, which is consisted of the knowledge uncertainty, evidence uncertainty, combinatorial evidence uncertainty, renewal of uncertainty, and conclusion uncertainty. The Bayesian model can simulate the causal relationship between real-world affairs by building a graphic model based on the graph theory and the probability theory; it is often used in precise reasoning algorithms such as polytree message passing algorithms, clique-tree message passing algorithms, graph reduction algorithms, and combinatorial optimization algorithms, etc.

\section{Design of the Bayesian Recommendation Model for the Course Selection System of Online Education Platforms}

\subsection{Architecture of the online course selection system}

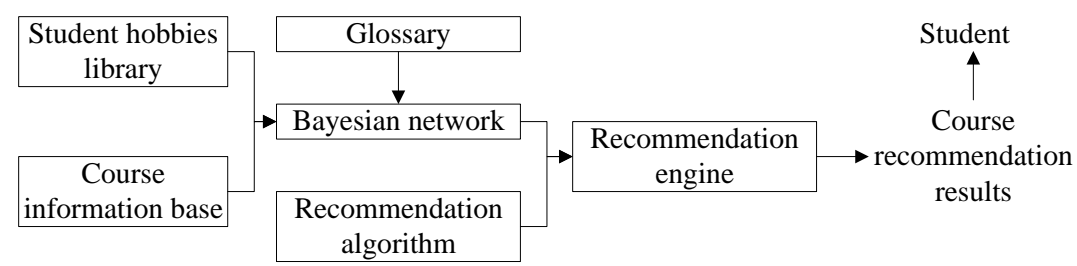

Fig. 4. System framework

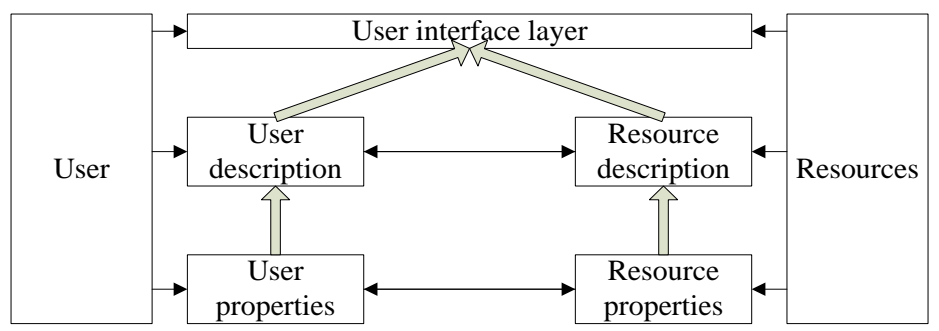

Fig. 5. Model of a rule-based system

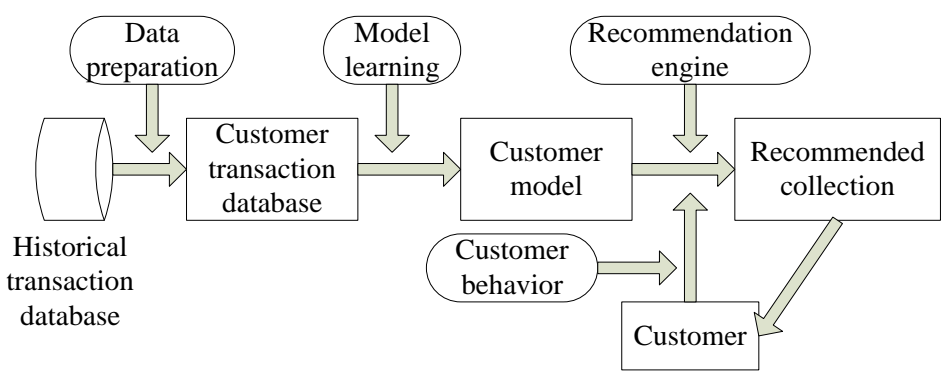

Fig. 6. Framework of the Bayesian network recommendation system 
The online course selection system mainly analyzes two kinds of information, the information of courses that are already selected by the student, and the information of courses that can be selected at present, and the courses that are already selected by the student can reflect the history information of the student's preferences. When constructing a Bayesian inference network of the relevant information, a recommendation engine was first designed based on the Bayesian network and corresponding recommendation algorithms, then the personalized recommendation results that meet the interests of students were given. Figure 4 shows the framework of the system. Taking the course selection of math major students as an example, the student preference library stores the preferences and weights characterized by the information of courses that are selected by all math major students; the course information library stores the information related to the courses; the glossary library stores the co-occurrence information between words in the glossary space, which is used to provide data support for the construction of Bayesian networks and the calculation of probabilities. Figure 5 shows the model of a rule-based system, which can be divided into three layers: description layer, keyword layer and user interface layer. Figure 6 shows the framework of the Bayesian network recommendation system. The framework includes the data preparation and transaction module, the pattern discovery modules, and the data set recommendation module, etc. According to the weights of the student's preferences, the recommendation engine combines with the student's course selection behaviors to generate a collection of recommended courses intelligently.

\subsection{Construction of Bayesian inference network for online course selection system}

Obtaining the model that can be used in data mining is the essential part of data preparation. Based on certain mining goals, some transactions that exist in the transaction set are generally quite important. Therefore, the process of making sparse transactions more compact can be regarded as a process of data expression. All nodes in the Bayesian inference network require a given condition probability table, since the nodes in the Bayesian network are divided into root nodes and non-root nodes, for nodes without a root node, their prior probabilities should be given, and the collection of the node condition probabilities is expressed as the condition probability table in the Bayesian network. Then, according to the known evidence nodes and the prior probabilities in the condition probability table, the posterior probability of the targe node to be queried could be calculated by the inference process of the Bayesian network. Figure 7 gives a diagram of the course recommendation process. After preferred courses and nodes are input, the system will automatically match the evidence nodes, then, according to the node probability distribution, it pushes a collection of recommended courses with the help of evidence dissemination.

Figure 8 shows the hierarchical structure of the data mining module, which is divided into an application layer, a data mining layer, and a basic algorithm layer. The data mining layer is mainly responsible for processing the information of courses that have already been selected by the student, and this information is taken as the input of the algorithm module, and it is the core of the data mining module. Figure 9 shows the 
flow of the course recommendation algorithm. By obtaining the basic information of the target student, calculating his/her interest in the courses based on history data of his/her learning behaviors, the vectors of user preference were constructed and clustered, in each cluster, according to the records of user courses, the pheromone of each course was calculated separately. After that, through calculation, a similar user set composed of $\mathrm{k}$ users with the highest similarity to the target user was obtained, according to which, the scores given by students on the courses were predicted, and then the heuristic values of the courses were calculated. At last, according to the score information and the course pheromone, the course recommendation probabilities were calculated and the recommended courses were generated.

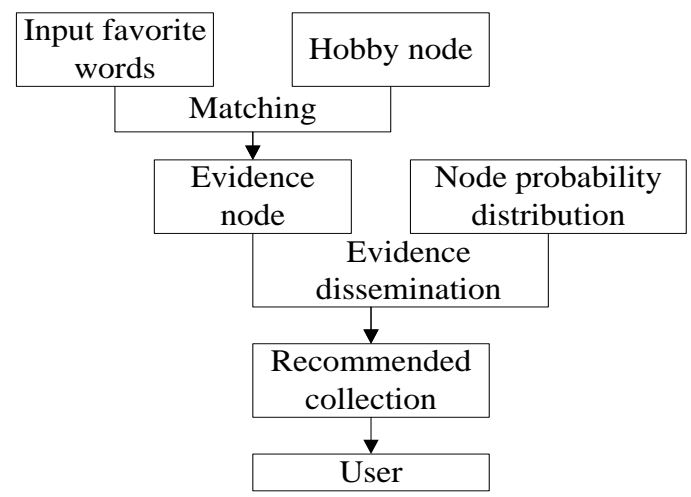

Fig. 7. Course recommendation process

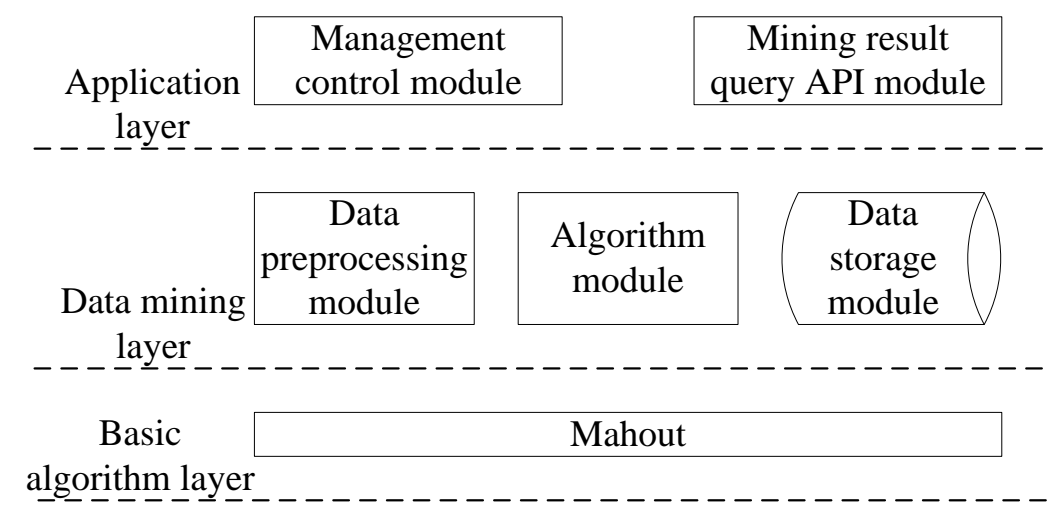

Fig. 8. The hierarchical structure of the data mining module 


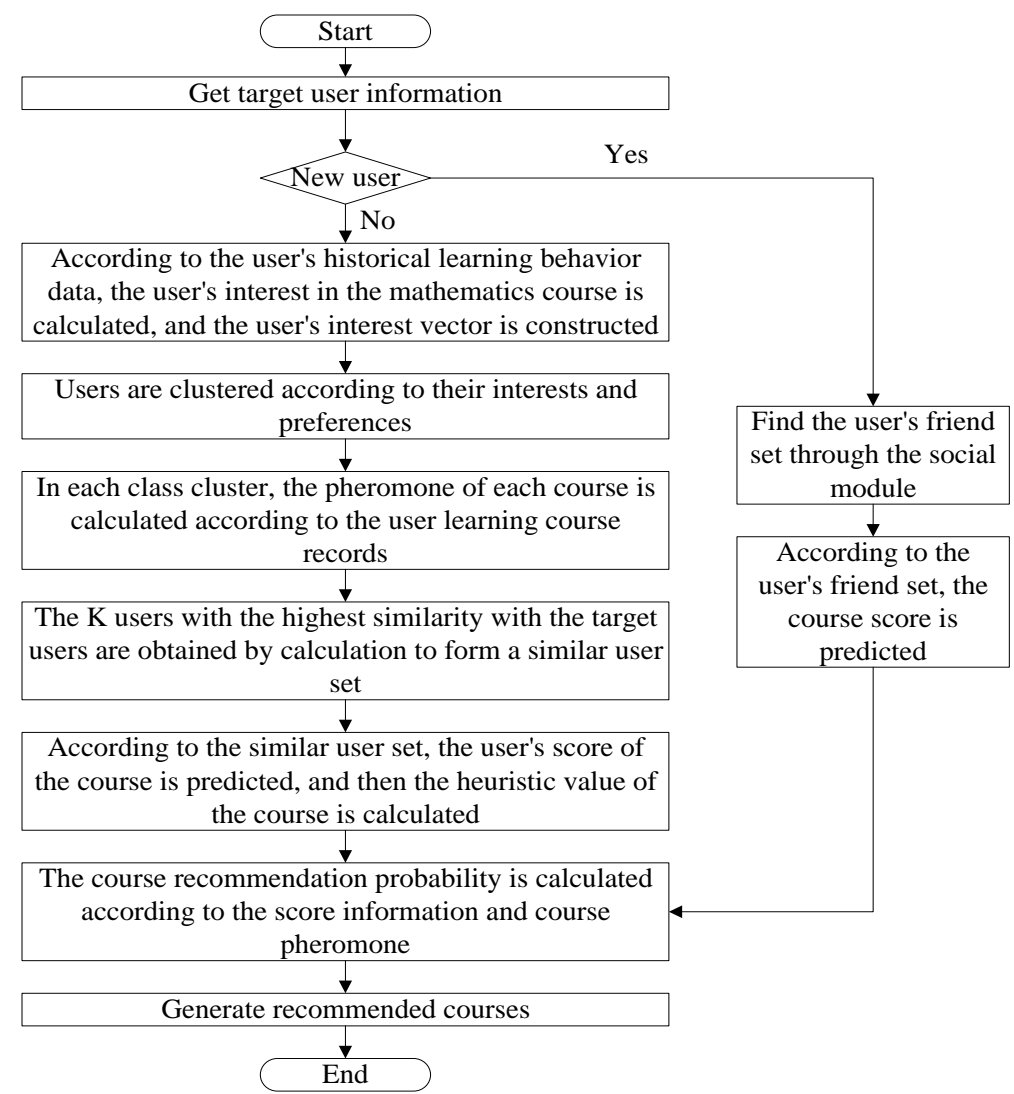

Fig. 9. Flow of the course recommendation algorithm

\section{Implementation and Analysis of the Automatic Optimal Course Recommendation System for Online Education Platforms}

\subsection{Implementation of the proposed system}

In traditional course selection systems, students can only search for the courses they want to take through keyword queries, in many cases, the matching of semantic concept of the input words cannot be guaranteed. However, with the help of the Bayesian inference network constructed in the previous chapter, after the students have selected the preference nodes, namely the system evidence of the Bayesian network is obtained, then, through evidence dissemination, the posterior probability values of the nodes related to the evidence can be obtained, each node calculates its own posterior probability based on the status information passed by the neighboring nodes and the condition probability table stored in the node. Figure 10 compares the 
mean absolute error under different numbers of optimal similar users. According to the figure, the number of similar users was between 10-80, the values of the mean absolute error of the Bayesian model and the collaborative filtering model were both within $[0.53,0.67]$, indicating that both algorithms had good score prediction accuracy. The two models roughly exhibited the same trend. The curve of the Bayesian model was flatter, indicating that the curve of the Bayesian model fluctuated greatly with the increase of the number of similar users.

The course selection score prediction test was performed using the Bayesian model, the user-based collaborative filtering model, the term-based collaborative filtering model, and the SlopeOne model, and the accuracy of the algorithms was compared. Figure 11 gives the score prediction results of the four algorithms. According to the figure, the mean absolute error and root mean square error of the Bayesian model were the lowest, indicating that it was significantly better than other models in predicting the course selection scores.

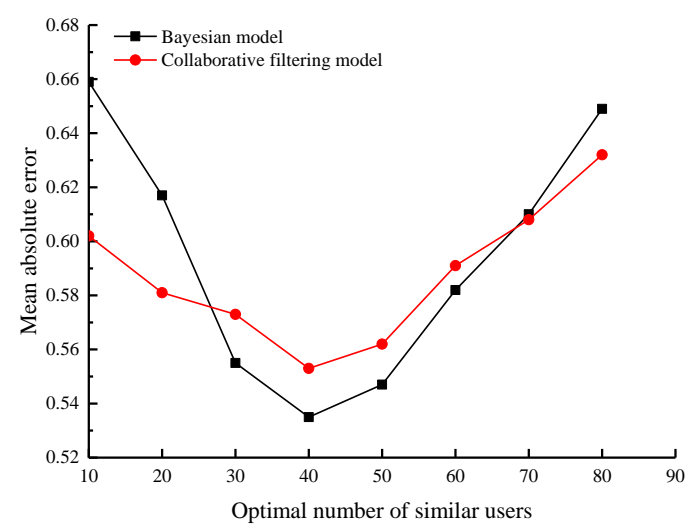

Fig. 10.Comparison of mean absolute error under different numbers of optimal similar users

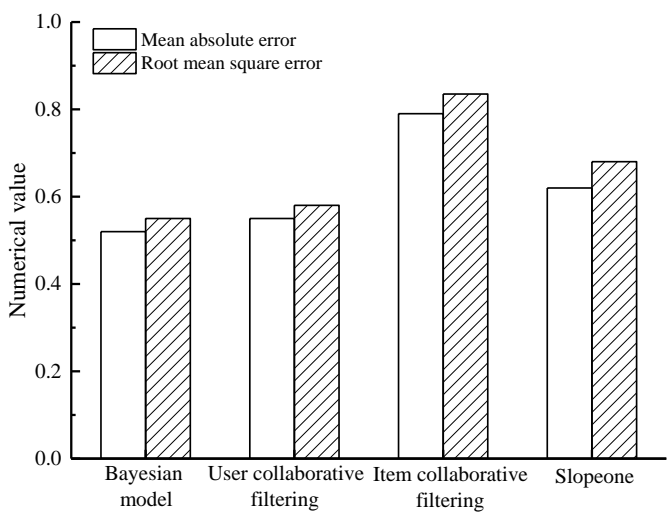

Fig. 11.Prediction results of four algorithms 


\subsection{Analysis of the proposed system}

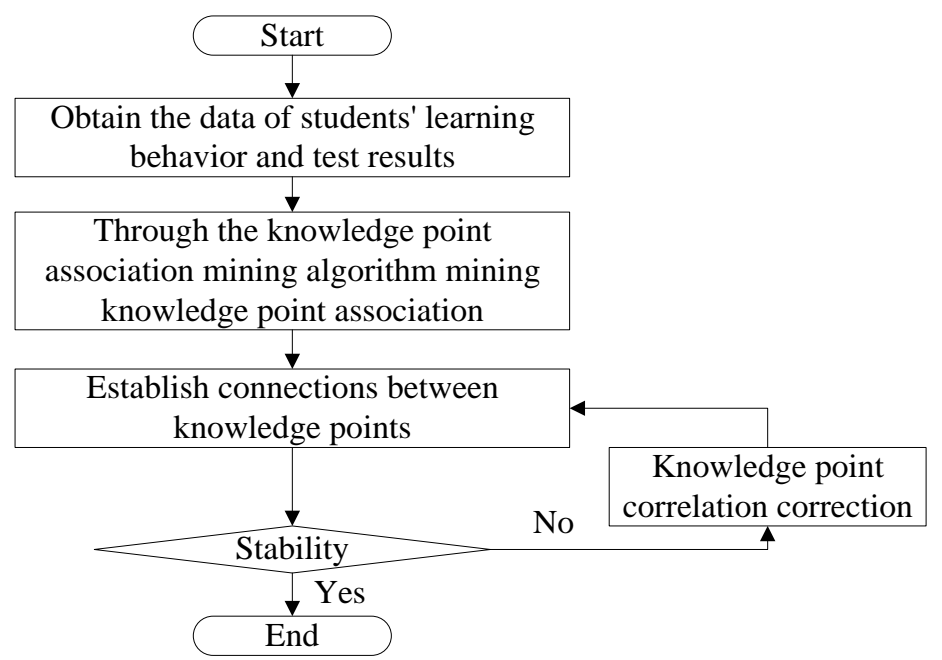

Fig. 12. A flowchart of knowledge point association mining

Course information is stored in the course information library, and each course has a detailed text introduction. For an evaluation system, a key concept of its retrieval performance is the "relevance", which can be used to determine the degree of the student's satisfaction with the optimal course selection scheme. Using the Bayesian model, the posterior probabilities of certain courses can be obtained based on the known preferences of the math major students, and they can be used to describe the recommendation degree of the courses. Figure 12 is a flowchart of knowledge point association mining. First, the user learning behavior and test result data are obtained, then the associations among knowledge points are mined by the knowledge point association mining algorithm to determine the knowledge point associations. In terms of accuracy, the course selection system established based on the matching method of the Bayesian model can greatly promote the course selection knowledge acquisition of math major students, using this system, students can select courses they preferred, since interest is the learning impetus for all people, under a better learning pattern, students would show greater interest in the courses. Moreover, even under the condition of multi-user course selection, the Bayesian model can still ensure a stable accuracy rate.

\section{Conclusion}

This paper explored an automatic optimal course recommendation method for online math education platforms based on the Bayesian model, and drew the following conclusions: 
1. The model can effectively merge priori and posteriori information, and encode the causality between knowledge points; by continuously adding information of new evidences, it can timely update the evaluation and judge the course selection.

2. All nodes in the Bayesian inference network require the given condition probability table, according to the known evidence nodes and the prior probabilities in the condition probability table, the posterior probability of the targe node to be queried could be calculated by the inference process of the Bayesian network.

3. The curve of the Bayesian model fluctuated greatly with the increase of the number of similar users. In terms of the prediction of course selection scores, the Bayesian model outperformed the user-based collaborative filtering model, the term-based collaborative filtering model, and the SlopeOne model.

4. Using the Bayesian model, the posterior probabilities of certain courses can be obtained based on the known preferences of the math major students, and they can be used to describe the recommendation degree of the courses, and the Bayesian model can ensure a stable accuracy rate.

\section{$6 \quad$ References}

[1] Es-Sajjade, A., Paas, F. (2020). Educational theories and computer game design: lessons from an experiment in elementary mathematics education. Educational Technology Research and Development, 68(5): 2685-2703. https://doi.org/10.1007/s11423-020-09799$\underline{\mathrm{w}}$

[2] DiSessa, A.A. (2018). Computational literacy and "the big picture" concerning computers in mathematics education. Mathematical Thinking and Learning, 20(1): 3-31. https://doi. org/10.1080/10986065.2018.1403544

[3] Leekitchwatana, P., Pimdee, P. (2020). An Analysisoff Thai Student Teacher Appropriate Internet Use Behaviour, International Journal of Emerging Technologies in Learning, 16(2): 254-271. https://doi.org/10.3991/ijet.v16i02.13747

[4] Xue, Y., Liu, Y., Ji, C., Xue, G. (2020). Hydrodynamic parameter identification for ship manoeuvring mathematical models using a bayesian approach. Ocean Engineering, 195: 106612. https://doi.org/10.1016/j.oceaneng.2019.106612

[5] Rui, G. (2019). Teaching physics in science, technology, engineering and mathematics education contexts with interactive computational modelling. AIP Conference Proceedings, 2116(1): 410002. https://doi.org/10.1063/1.5114426

[6] Rui, G. (2019). Preface of the "symposium on computers, modelling and interactive environments in science, technology, engineering and mathematics education". AIP Conference Proceedings, 2116(1): 410001. https://doi.org/10.1063/1.5114425

[7] Murchan, D., Oldham, E. (2017). Exploring the role of computer-based assessment in diagnosing children's mathematical errors in primary education in ireland. Irish Educational Studies, 36(2): 489-510. https://doi.org/10.1080/03323315.2017.1393765

[8] Chefrour, A., Souici-Meslati, L., Difi, I., Bakkouche, N. (2019). A novel incremental learning algorithm based on incremental vector support machina and incremental neural network learn++. Revue d'Intelligence Artificielle, 33(3): 181-188. https://doi.org/10.182 80/ria.330303

[9] Singh, G., Agrawal, S., Sohi, B.S. (2020). Handwritten Gurmukhi digit recognition system for small datasets. Traitement du Signal, 37(4): 661-669. https://doi.org/10.18280/ts.370 $\underline{416}$ 
[10] Moeller, K., Fischer, U., Nuerk, H.C., Cress, U. (2015). Computers in mathematics education - training the mental number line. Computers in Human Behaviour, 48: 597607. https://doi.org/10.1016/j.chb.2015.01.048

[11] Nagata, K., Nakamura, T., Farouk, A. (2017). Quantum cryptography based on the Deutsch-Jozsa algorithm. International Journal of Theoretical Physics, 56(9): 2887-2897. https://doi.org/10.1007/s10773-017-3456-X

[12] Heidari, S., Naseri, M., Gheibi, R., Baghfalaki, M., Pourarian, M. R., Farouk, A. (2017). A new quantum watermarking based on quantum wavelet transforms. Communications in theoretical Physics, 67(6): 732. https://doi.org/10.1088/0253-6102/67/6/732

[13] Cheng, X., Zhao, C.Y. (2019). Prediction of tourist consumption based on Bayesian network and big data. Ingénierie des Systèmes d'Information, 24(5): 491-496. https:// doi.org/10.18280/isi.240505

[14] Akbar, S., Midhunchakkaravarthy, D. (2020). A novel filtered segmentation-based Bayesian deep neural network framework on large diabetic retinopathy databases. Revue d'Intelligence Artificielle, 34(6): 683-692. https://doi.org/10.18280/ria.340602

[15] Bulinski, Z., Orlande, H., Krysinski, T., Werle, S., Ziolkowski, L. (2019). Coupled podbayesian estimation of the parameters of mathematical model of the packed-bed drying of cherry stones. Energy, 181: 345-359. https://doi.org/10.1016/j.energy.2019.05.166

[16] Maclean, S., Labahn, G. (2014). A bayesian model for recognizing handwritten mathematical expressions. Pattern Recognition, 48(8): 2433-2445. https://doi.org/10.1016 /j.patcog.2015.02.017

[17] Batle, J., Ciftja, O., Naseri, M., Ghoranneviss, M., Farouk, A., \& Elhoseny, M. (2017). Equilibrium and uniform charge distribution of a classical two-dimensional system of point charges with hard-wall confinement. Physica Scripta, 92(5): 055801. https://doi.org/1 $\underline{0.1088 / 1402-4896 / a a 6630}$

[18] Tezer, M., Kanbul, S. (2009). Opinions of teachers about computer aided mathematics education who work at special education centres. Procedia-Social and Behavioural Sciences, 1(1): 390-394. https://doi.org/10.1016/j.sbspro.2009.01.070

[19] Chuang, H.C. (2015). Mathematical modelling and bayesian estimation for error-prone retail shelf audits. Decision Support Systems, 80: 72-82. https://doi.org/10.1016/j.dss.20 $\underline{15.10 .003}$

[20] Enright, C.G., Madden, M.G., Madden, N. (2013). Bayesian networks for mathematical models: techniques for automatic construction and efficient inference. International Journal of Approximate Reasoning, 54(2): 323-342. https://doi.org/10.1016/j.ijar.2012.10.004

[21] Bulygina, N., Gupta, R. (2010). How bayesian data assimilation can be used to estimate the mathematical structure of a model. Stochastic Environmental Research and Risk Assessment, 24(6): 925-937. https://doi.org/10.1007/s00477-010-0387-y

[22] Ruthven, K. (2002). Instrumenting mathematical activity: reflections on key studies of the educational use of computer algebra systems. International Journal of Computers for Mathematical Learning, 7(3): 275-291. https://doi.org/10.1023/A:1022108003988

[23] Mac'kowski, M.S., Brzoza, P.F., Spinczyk, D.R. (2018). Tutoring math platform accessible for visually impaired people. Computers in Biology and Medicine, 95: 298-306. https://doi.org/10.1016/j.compbiomed.2017.06.003

[24] Ohanu, I.B., Chukwuone, C.A. (2018). Constraints to the use of online platform for teaching and learning technical education in developing countries. Education \& Information Technologies, 23(6): 3029-3045. https://doi.org/10.1007/s10639-018-9757-8

[25] Torous, J., Grunau, Z., Thom, R., Boland, R., Meyer, F. (2018). Connecting through technology: a collaborative psychiatry trainee and educator online platform. Academic Psychiatry, 42(3): 426-427. https://doi.org/10.1007/s40596-018-0910-x 
[26] Kim, D., Lee, Y., Leite, W.L., Huggins-Manley, A.C. (2020). Exploring student and teacher usage patterns associated with student attrition in an open educational resourcesupported online learning platform. Computers \& Education, 156: 103961. https://doi.org/ 10.1016/j.compedu.2020.103961

[27] Ramin, M., Arhonditsis, G.B. (2013). Bayesian calibration of mathematical models: optimization of model structure and examination of the role of process error covariance. Ecological Informatics, 18: 107-116. https://doi.org/10.1016/j.ecoinf.2013.07.001

[28] Wen, J., Wei, X., He, T., Zhang, S. (2020). Regression analysis on the influencing factors of the acceptance of online education platform among college students. Ingénierie des Systèmes D Information, 25(5): 595-600. https://doi.org/10.18280/isi.250506

[29] Zhu, Y., Liu, D., Chen, G., Jia, H., Yu, H. (2013). Mathematical modelling for active and dynamic diagnosis of crop diseases based on Bayesian networks and incremental learning. Mathematical and Computer Modelling, 58(3-4): 514-523. https://doi.org/10.1016/j.ijcar d.2018.07.065

[30] Kolluru, S., Varughese, J.T. (2017). Structured academic discussions through an online education-specific platform to improve pharm.d. students learning outcomes. Curr Pharm Teach Learn, 9(2): 230-236. https://doi.org/10.1016/j.cptl.2016.11.022

[31] Ferrin, E.H., Pratt, A., Jona, K. (2013). A remote online microscopy lab as a tool for k-12 science education and a platform for teacher-scientist partnerships. Microscopy \& Microanalysis, 19(S2): 292-293. https://doi.org/10.1017/S1431927613003450

[32] Zeng, Q., Zhong, X. (2012). The design of individual knowledge sharing platform based on blog for online information literacy education. Physics Procedia, 33: 1426-1432. https://doi.org/10.1016/j.phpro.2012.05.233

\section{Authors}

Yongyan Fan, graduated from Hebei University in 2009. Worked in Cangzhou Normal University. Her research interests include differential equations and boundary value problems. dttongcz@163.com

Dingli $\mathbf{Z u}$ is an associate professor working on applied mathematics in the department of mathematics and physics in Chengde Petroleum College, China, His research interests include ordinary differential equation, partial differential equation and mathematics teaching. zzzjjij4104@163.com

Jing Zhang, graduated from Hebei University of Technology in 2011. Worked in Cangzhou Normal University. Her research interests include operations research and cybernetics. z770477@126.com .

Hongyu Zhang, graduated from Chengdu University of information Engineering in 2003. Worked in Chengde petroleum college. His research focus on the teaching of mathematics and mathematical modelling. z1234561979@126.com .

Article submitted 2021-05-03. Resubmitted 2021-06-04. Final acceptance 2021-06-05. Final version published as submitted by the authors7. 